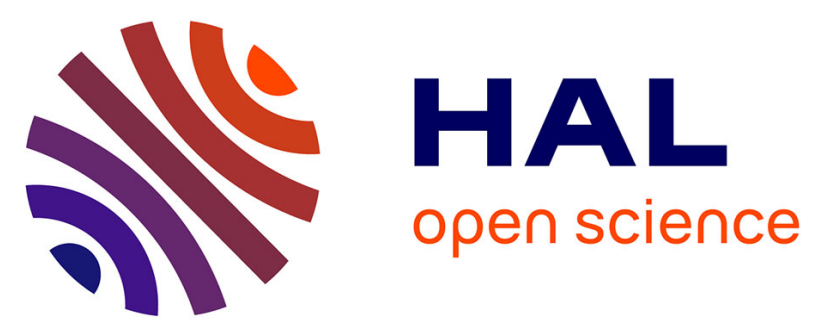

\title{
Spatial integration of baseline-free damage detection algorithms based on dual-PZT for the structural health monitoring of anisotropic composite aeronautic structures
}

Emmanuel Lize, Marc Rebillat, Nazih Mechbal, Christian Bolzmacher

\section{To cite this version:}

Emmanuel Lize, Marc Rebillat, Nazih Mechbal, Christian Bolzmacher. Spatial integration of baselinefree damage detection algorithms based on dual-PZT for the structural health monitoring of anisotropic composite aeronautic structures. International Workshop on Structural Health Monitoring 2019, Sep 2019, Palo Alto, United States. pp.1-8, 10.12783/shm2019/32386 . hal-03013029

\author{
HAL Id: hal-03013029 \\ https://hal.science/hal-03013029
}

Submitted on 18 Nov 2020

HAL is a multi-disciplinary open access archive for the deposit and dissemination of scientific research documents, whether they are published or not. The documents may come from teaching and research institutions in France or abroad, or from public or private research centers.
L'archive ouverte pluridisciplinaire HAL, est destinée au dépôt et à la diffusion de documents scientifiques de niveau recherche, publiés ou non, émanant des établissements d'enseignement et de recherche français ou étrangers, des laboratoires publics ou privés. 


\title{
Spatial integration of baseline-free damage detection algorithms based on dual-PZT for the structural health monitoring of anisotropic composite aeronautic structures
}

\author{
Emmanuel LIZÉ, Marc RÉBILLAT², Nazih MECHBAL ${ }^{2}$, Christian BOLZMACHER ${ }^{1}$ \\ 1 CEA, LIST, Sensorial and Ambient Interfaces Laboratory, 91191 - Gif-sur-Yvette CEDEX, France. \\ 2 Processes and Engineering in Mechanics and Materials Laboratory (PIMM, CNRS-CNAM-ENSAM-HESAM Paris, France)
}

\begin{abstract}
The focus is put here on the Structural Health Monitoring (SHM) of composite aeronautic structure using Lamb waves emitted and recorded with piezoelectric transducers (PZT). Conventional algorithms perform Lamb waves acquisition in the healthy state of the structure (referred to as the "baseline") and then compare incoming data from an unknown state with this one to detect, locate, classify and quantify any potential damage. The acquisition, storage, and update of the initially recorded baseline database constitute a severe drawback of such algorithms. Indeed, the structure under study as well as the environment may vary during its operational life without the appearance of any damage and thus the initial baseline may not be relevant at any instant where damage monitoring is needed. In order to circumvent this drawback, "baselinefree" method (such as the instantaneous baseline [BI] and rupture of reciprocity [RR]) have been developed. Moreover, the use of dual-PZT, i.e. concentric PZT made of a ring and a disk lying on the same ceramic, has been shown as attractive for baselinefree purposes. However, now that several algorithms based on dual-PZT are available, no study dealing with the spatial integration of the results provided by these algorithms have been reported in the literature. It is thus proposed in this paper to investigate strategies for the spatial integration of common baseline-free methods (namely BI and $\mathrm{RR}$ ) on an experimental case of damage on a highly anisotropic composite plate. Results illustrate the decomposition of Lamb wave modes in signals measured via dual PZTs as well as the proposed spatial integration strategies for these methods.
\end{abstract}

\section{INTRODUCTION}

Monitoring in real-time and autonomously the health state of structures is of high interest in the industry, and more specifically for the aeronautic and civil engineering applications fields. Such a process is referred to as Structural Health Monitoring (SHM) [1, 2]. To achieve this goal, these structures become "smart" in the sense that they are equipped with sensors, actuators and artificial intelligence that allow them to state regarding their own health. One can compare such smart structures with the human body which, thanks to its various senses and nerves, can know if it has been hurt and where. The SHM process is classically decomposed into four steps: damage detection, damage localization, damage classification and damage quantification.

Damage detection using Lamb waves is one of the most common methods for the SHM of aeronautic composite structures. Among all the methods employed to trigger and catch Lamb waves, the use of piezoelectric transducer (PZT) is one of the cheapest and easiest-to-settle. The common approach is based on the comparison of signals recorded on a "pristine" structure - the baseline - against those obtained on a possibly damaged structure. The resulting signal strongly depends on the structure's mechanical and geometrical properties as well as environmental conditions. Some 
solutions have been proposed to compensate for the effect of temperature $[3,4]$ or to extend baseline data to a wider range of environmental conditions $[5,6]$. This drawback of existing methods has also fed the interest for "baseline-free" methods. "Baselinefree" is here an abusive word (see Axiom II in [1]) since data, models, or physical assumptions are always needed to decide whether a structure is damaged or not. This expression thus refers here to methods where a baseline of signals recorded from the pristine condition of the monitored structure is not mandatory for damage detection.

Lamb wave excitation in composite aeronautic structures generates simultaneously at least two propagating modes having different physical properties (velocity, wave number, etc...). Those symmetric and antisymmetric Lamb wave modes are very useful for SHM as they convey complementary information when interacting with damages [6]. Dual PZTs are made of a concentric ring and disc. Both parts can be used as actuators and sensors bringing more signal combinations than conventional PZTs and allow for the isolation of the first antisymmetric $A_{0}$ or symmetric $S_{0}$ mode [7, 8]. With dual PZTs, damage can then be detected in a "baselinefree" manner by using the damage-introduced mode conversion and attenuation of the propagating waves. The theoretical aspect justifying the use of those transducers for "baseline-free" SHM is well developed in and experiments [7].

Svral "baseline-free" methods, where mode decomposition based on dual PZT have been proposed. The "instantaneous baseline" (BI) method is based on a transducer network with paths having the same dimensions and orientations. Instead of comparing signals obtained at a random condition with a pristine baseline, signals are compared instantaneously between paths even under varying environmental conditions $[9,10,11]$. Drawbacks are that examined paths must have identical PZT positioning and bonding and that reflection of elastic waves from boundaries must be avoided. In the "reciprocity principle" (RR) method, two signals obtained from both possible directions of a given path is directly compared. Its drawbacks are that the implemented PZTs must be identically bonded to the structure, and that damages located far from the investigated path are difficult to detect [12].

However, even if these "baseline-free" algorithms based on dual-PZT are available, no study dealing with the spatial integration of the results provided by these algorithms have been reported in the literature. Drawing on previous works of the authors [13], it is thus proposed in this paper to investigate strategies for the spatial integration of common baseline-free methods (namely BI, RR) on an experimental case of damage on a highly anisotropic composite plate.

The outline of the paper is as follows: in section 2 the structure under study mounted with dual PZTs is presented. The two proposed baseline-free damage detection approaches are described in section 3 . Then in section 4 , four methods dedicated to the computation of a damage localization map from the previously computed damage indexes are investigated. Results are presented, analyzed and discussed in section 5.

\section{STRUCTURE UNDER STUDY}

The structure under study is a CFRP plate, with plies having $\left[0^{\circ} 90^{\circ}\right]_{16}$ stacking and properties as shown in Figure 1. This plate is equipped with 7 dual-PZTs produced by NOLIAC (NCE51 material). 


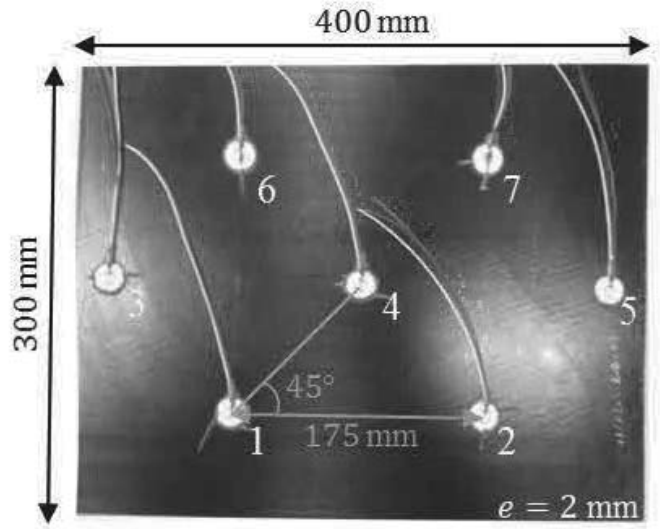

(a)

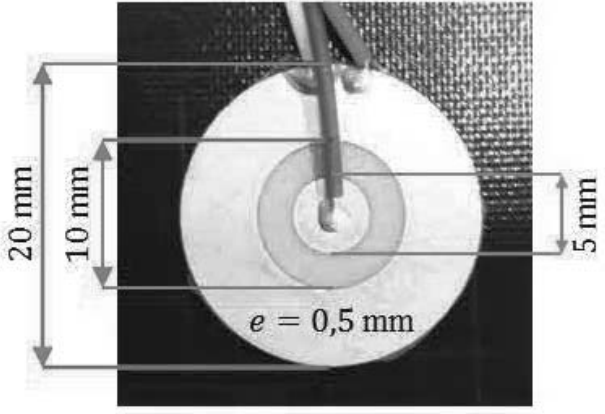

(b)

Figure 1 : a) Dimensions of the $\left[0^{\circ} 90^{\circ}\right]_{16}$ CFRP plate and dual-PZTs positions. b. Dimensions of dual-PZTs.

The delamination-type damage is introduced into samples in a calibrated way using Laser Shock Wave Testing (LWST) and more particularly symmetrical laser shock configuration. LSWT is chosen as an alternative to conventional damage generation techniques such as conventional impacts and Teflon inserts since it allows for a better calibration of damage in type, depth and size. In the present case a $20 \mathrm{~mm}$ diameter damage has been created by cumulating damages produced by successive and close LASER shocks. The delamination control has been carried out using standard C-Scan and results demonstrate that the CFRP plate is indeed damaged as the required position. However, a visual inspection of the plate surface does not allow to detect any damage.

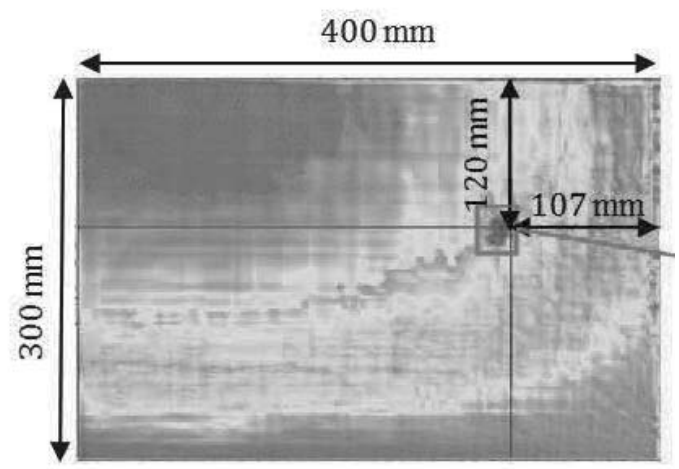

Figure 2: C-Scan of the damaged plate after multiple LASER Shocks. Visual inspection of the plate does not allow distinguishing the damaged area.

Damage indexes, or features, are then computed on the basis of the experimental signals ( 5 cycle sinusoidal tone burst modulated by a Hanning window at an amplitude of $10 \mathrm{~V}$ and a central frequency $f_{0}=50 \mathrm{kHz}$ ) after denoising. A damage index corresponding to the different between the maximum amplitude of the envelope of the two compared signals is computed for each path of the structure.

\section{BASELINE FREE METHODS UNDER STUDY}

Two "baseline-free » methods are here investigated using the damage index computed on the previously described structure under study. 


\section{a. Reciprocity principle}

This method has originally been implemented with dual-PZTs for crack detection within aluminum structures and with standard PZT elements in a composite structure. Its extension to composite structures equipped with dual PZTs allows to study the impact of delamination on each mode. In this case, the two signals at the basis of the damage indexes computation are $V_{a s-A B}$ et $V_{a s-B A}$ (the as index depicts the chosen actuating/sensing part of the dual-PZTs [disk, ring, entire], and the index $A B$ refers to the dual PZTs actuator sensor configuration). This method can naturally be used by considering isolated $A_{0}$ and $S_{0}$ modes and in these cases, the signals used for damage index computations are denoted $V_{a s-A B}^{A_{0}}$ and $V_{a s-B A}^{A_{0}}$ or $V_{a s-A B}^{S_{0}}$ and $V_{a s-B A}^{S_{0}}$ which correspond to symmetric and antisymmetric mode contributions extracted from the previous signals.

\section{b. Instantaneous baseline}

This method is the most mature one and the most widely used. Isolation of $A_{0}$ and $S_{0}$ modes within this method should allow to improve damage detection in several situations. For this method, the experimental signals used for damage index computation corresponds to the signal recorded one path and to the reference signal which is the average signal overall comparable paths. Comparable paths existing on the structure under study are depicted in the following Figure. This method can naturally be used by considering isolated $A_{0}$ and $S_{0}$ modes and in these cases, the signals used for damage index computation correspond to symmetric and antisymmetric mode contributions extracted from the previously defined signals.
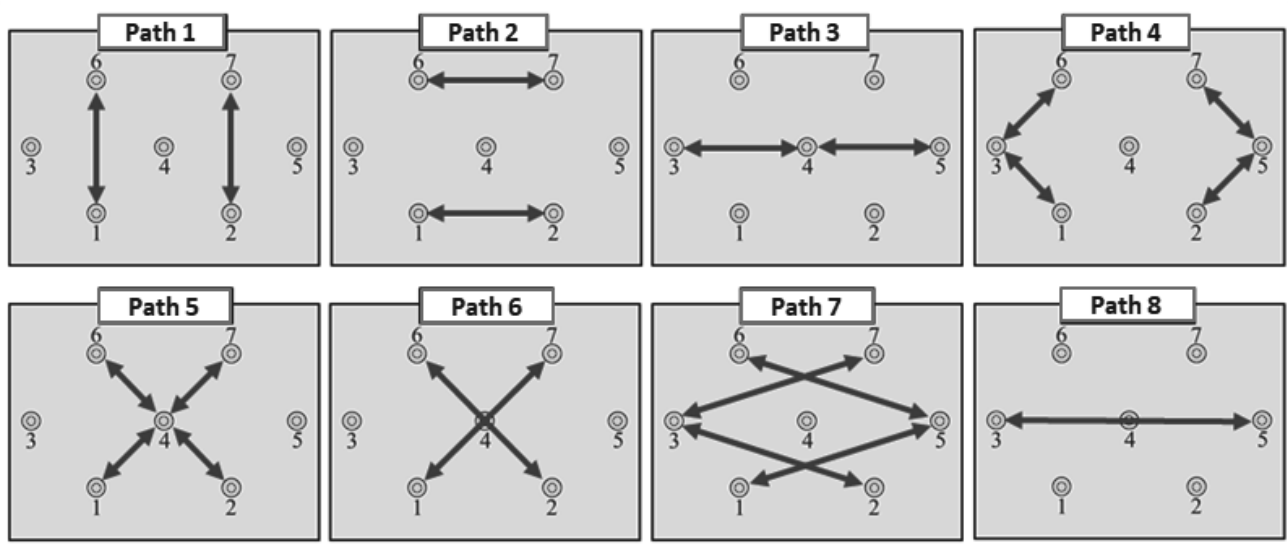

Figure 3: Comparable paths for the instantaneous baseline method

\section{DAMAGE LOCALIZATION MAPS COMPUTATION}

Four methods dedicated to the computation of damage localization map from the previously computed damage indexes are here investigated. They are denoted as "PATH, PZT, PIXEL, and CLUSTER". These methods constitute various potential ways to integrate damage indexes over the surface of the structure under study. 

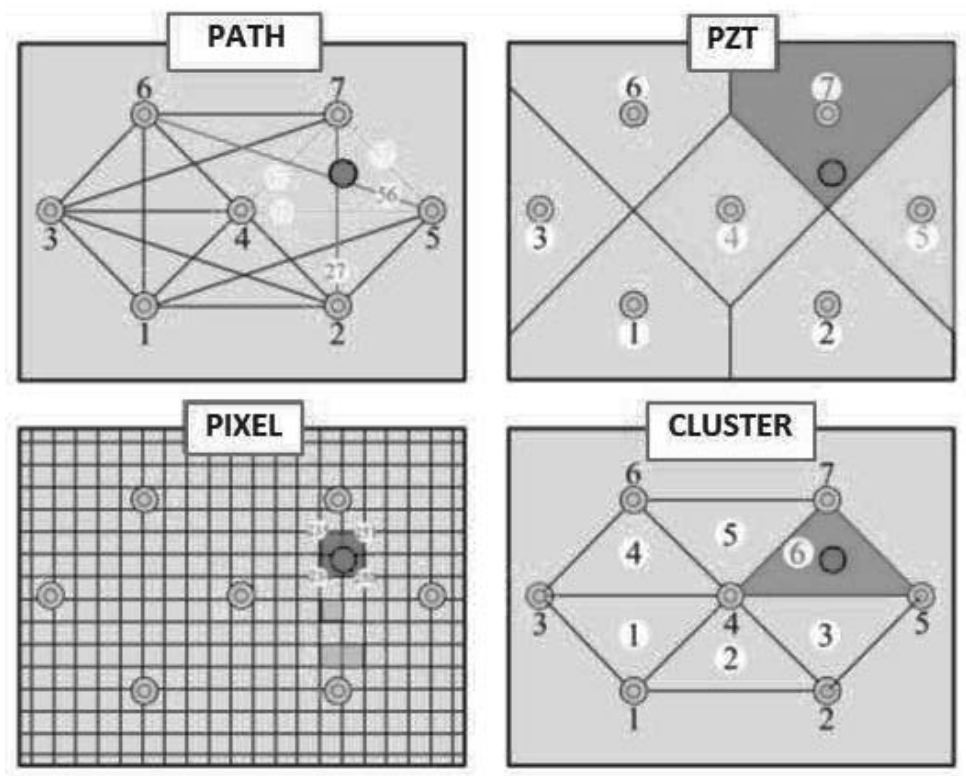

Figure 4: Tested strategies for damage indexes integration over the structures under study.

The "PATH" approach relies on paths comparison over the whole structure. For the structure under study, containing 7 dual-PZTs, 42 paths can be defined for which a damage index is computed. The "PZT" approach relies on the computation of one damage index per PZT by considering the average damage index over all the paths including that PZT. As the structure under study contains 7 PZT, 7 of such damage indexes can be computed that represent the damage state in the vicinity of each dualPZT. Areas associated with each dual-PZTs have been obtained through a Voronoi partitioning algorithm. Within the "PIXEL" approach the structure under study is divided arbitrarily in square zones, denoted as "PIXELs", according to the experimenter choice. One PIXEL stands for a zone delimited by this mesh. For each PIXEL, the computed damage index (DI) value corresponds to the mean of the DI values related to paths that cross that PIXEL divided by the number of such paths. For the structure under study, square PIXELs of side size of $20 \mathrm{~mm}$ have been retained and thus 336 PIXELs have been defined. Within the CLUSTER approach, a CLUSTER denotes an area delimited by PZT elements, as shown in Figure 4. One DI is computed for each CLUSTER by considering the DIs over the paths crossing the CLUSTER. The structure under study has been divided in 6 of such CLUSTERs as shown above.

\section{RESULTS}

Examples of results obtained when applying the previously defined baseline-free methods with the various spatial integration procedures and by making use of either S0 or A0 modes are presented.

In Figure 5, results for the reciprocity method, using the S0 mode and spatially integrated using the PZT approach are presented. From this figure, it can be seen that DIs do not vary largely over the various PZT. Furthermore, the maximum DI value is reached for PZT 6, which is neighboring PZT 7 corresponding to the PZT closest to the damaged area. That combination of methods (reciprocity rupture / S0 mode / PZT approach) is thus not really successful in the present example. 

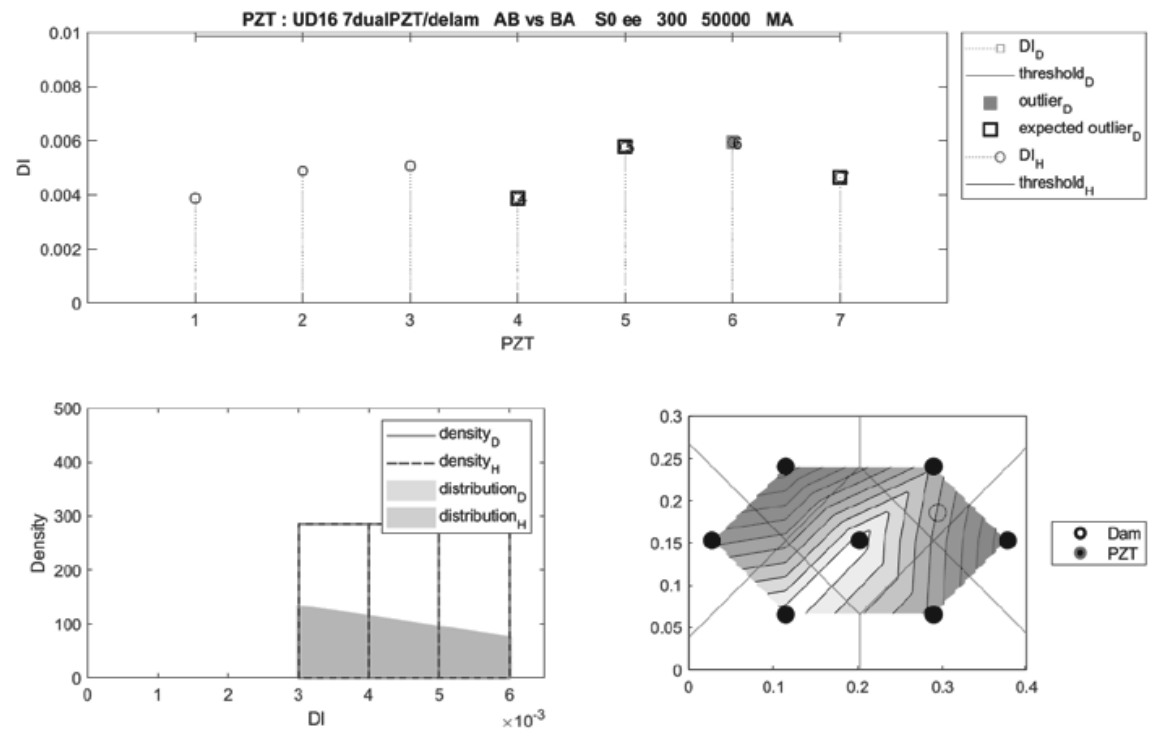

Figure 5: Reciprocity rupture method for the SO mode integrated with the PZT approach
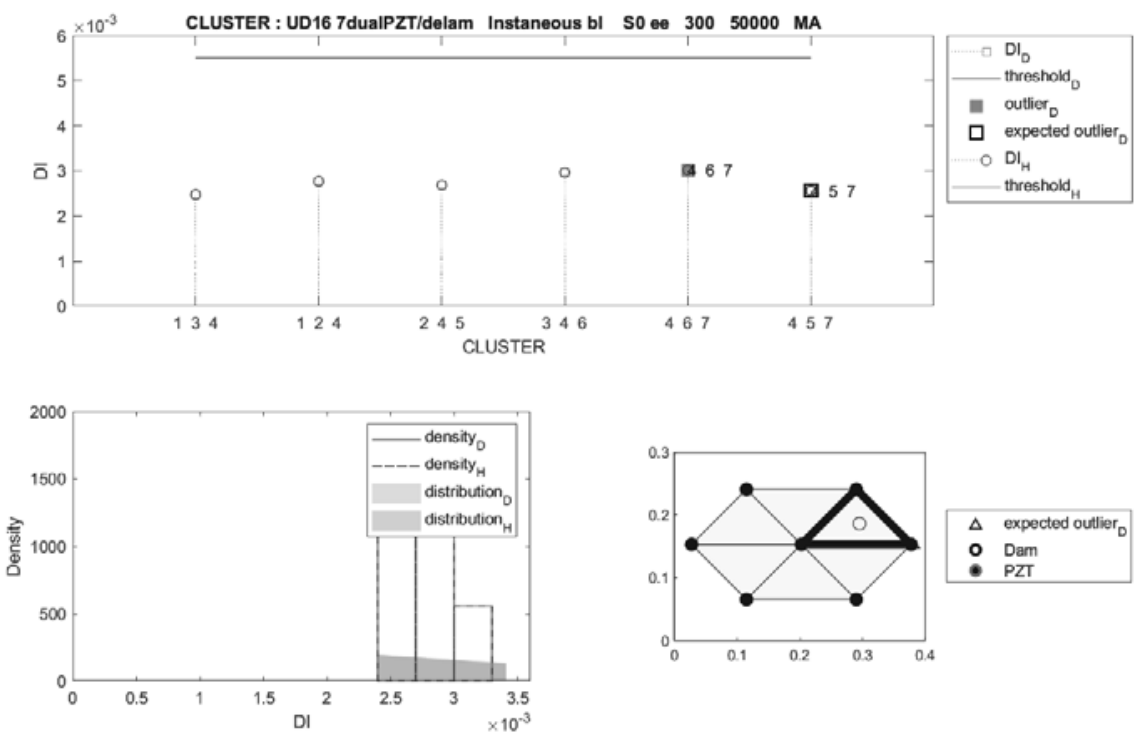

Figure 6: Instantaneous baseline method for the SO mode integrated with the CLUSTER approach

In Figure 6, results for the instantaneous baseline method, using the S0 mode and spatially integrated using the CLUSTER approach are presented. From this figure, damage indexes again do not vary largely over the various CLUSTERs. Furthermore, the maximum damage index value is reached for CLUSTER (4/6/7), which is neighboring CLUSTER (4/5/7) which contains the damaged area. Again, that combination of methods (instantaneous baseline / S0 mode / CLUSTER approach) is not really successful in the present example. 

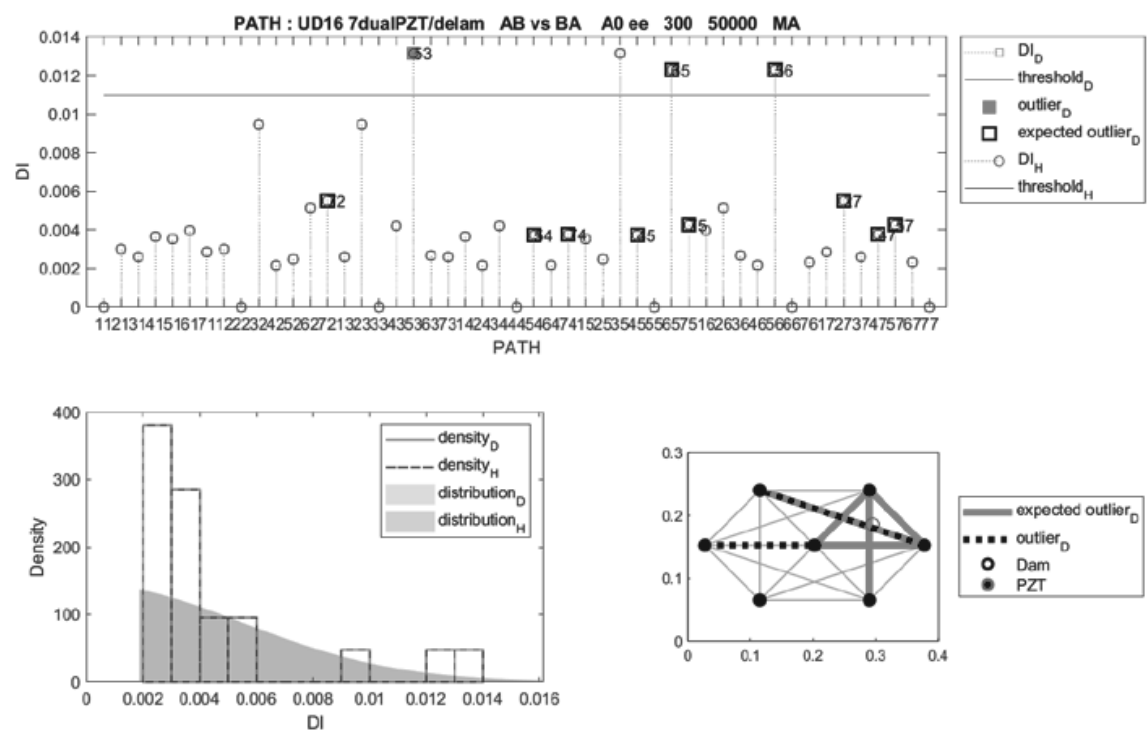

Figure 7: Reciprocity rupture method for the AO mode integrated with the PATH approach

In Figure 7, results for the reciprocity rupture method, using the A0 mode and spatially integrated using the PATH approach are presented. From this figure, it can be observed that DIs vary largely over the various PATHs. Furthermore, the maximum damage index value is reached for several PATHs crossing, or very close to the damaged area. That combination of methods (reciprocity rupture / A0 mode / PATH approach) is here successful in the present example.
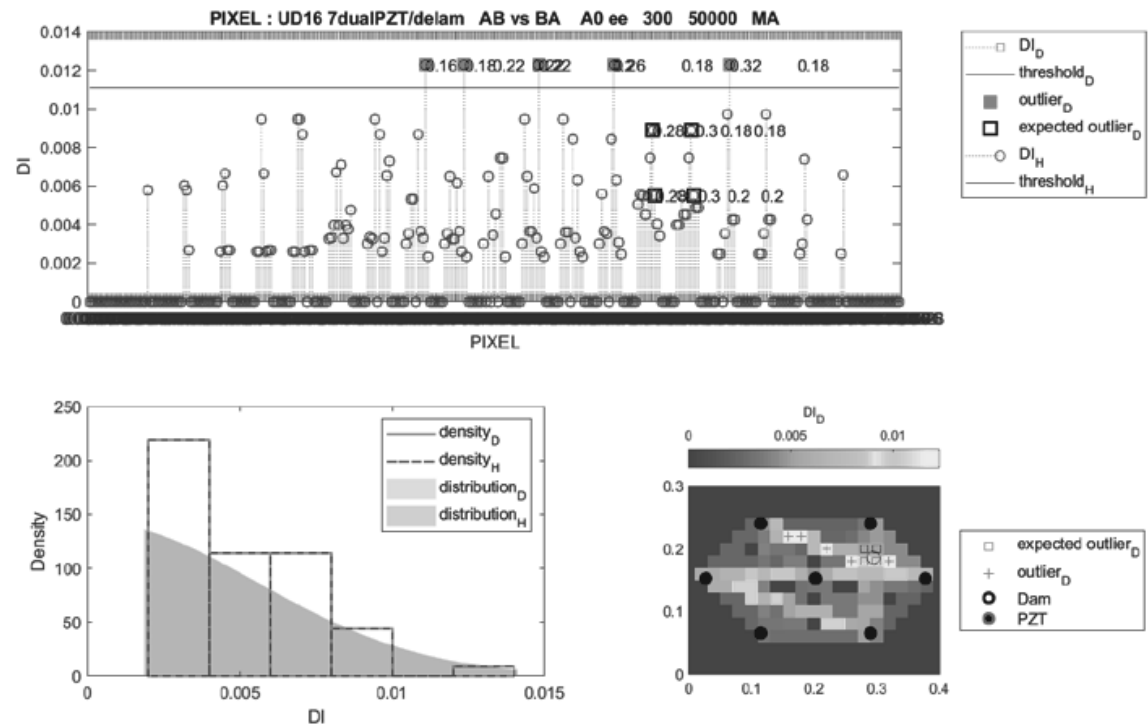

Figure 8: Reciprocity rupture method for the AO mode integrated with the PIXEL approach

In Figure 8, results for the reciprocity rupture method, using the A0 mode and spatially integrated using the PIXEL approach are presented. From this figure, it can be observed that damage indexes again vary largely over the various PATHs. Furthermore, the maximum damage index value is reached for several PIXELs containing, or very close 
to the damaged area. That combination of methods (reciprocity rupture / A0 mode / PIXEL approach) is here again successful in the present example.

\section{CONCLUSION}

In order to circumvent the drawback of standard SHM methods, F"baseline-free" method (such as the instantaneous baseline (BI) and rupture of reciprocity (RR)) have been developed. The use of dual-PZT, i.e. concentric PZT made of a ring and a disk lying on the same ceramic, has also been shown as attractive for baseline-free purposes. This paper deals with the spatial integration of the results provided by these algorithms. Four strategies for the spatial integration of common baseline-free methods (namely BI and RR) are illustrated on an experimental case of damage on a highly anisotropic composite plate. Results illustrate that the proposed spatial integration strategies for these methods may be relevant in practice.

\section{REFERENCES}

[1] J. M. Nichols et M. D. Todd, «Encyclopedia of Structural Health Monitoring,» John Wiley \& Sons, Ltd, 2009.

[2] W. Staszewski, C. Boller et G. R. Tomlinson, Health Monitoring of Aerospace Structures: Smart Sensor Technologies and Signal Processing, First éd., Wiley, 2004.

[3] C. Fendzi, N. Mechbal, M. Rebillat, M. Guskov et G. Coffignal, «A general Bayesian framework for ellipse-based and hyperbola-based damage localization in anisotropic composite plates,» Journal of Intelligent Material Systems and Structures, vol. 27, pp. 350-374, 2016.

[4] Y. Zheng, C. Martinez, D. Easton, G. Park et K. Farinholt, «Sensor Self-Diagnostics for Piezoelectric Transducers Operating in Harsh Temperature Environments,» chez Proceedings of SPIE Smart Structures and Materials \& Nondestructive Evaluation and Health Monitoring Conference, San Diego CA, 2011.

[5] C. Liu, J. B. Harley, M. Bergés, D. W. Greve et I. Oppenheim, «Robust ultrasonic damage detection under complex environmental conditions using singular value decomposition,» Ultrasonics, vol. 58, $\mathrm{n}^{\circ} \% 175-86,2015$.

[6] R. Hajrya et N. Mechbal, «Principal component analysis and perturbation theory based robust damage detection of multifunctional aircraft structure,» Structural Health Monitoring - An International Journal, vol. 12, pp. 263-277, 2013.

[7] Z. Su et L. Ye, Identification of Damage Using Lamb Waves, Springer-Verlag London, 2009.

[8] C. Yeum, H. Sohn et J. B. Ihn, «Delamination detection in a composite plate using a dual piezoelectric transducer network,» Proc. SPIE, 2011.

[9] C. Yeum, H. Sohn et J. B. Ihn, «Lamb wave mode decomposition using concentric ring and circular piezoelectric transducers,» Wave Motion, vol. 48, p. 358-70, 2011.

[10] H. J. Lim, H. Sohn, C. Yeum et J. M. Kim, «Reference-free damage detection, localization, and quantification in composites,» Journal of Auoust. Soc. Am. , vol. 133, p. quantification in, 2013.

[11] Y.-K. An et H. Sohn, «Instantaneous crack detection under varying temperature and static loading conditions,» Structural Control and Health Monitoring, vol. 17, p. 730-41, 2010.

[12] S. R. Anton, D. J. Inman et G. Park, «Reference-free damage detection using instantaneous baseline measurements,» AIAA Journal, vol. 47, pp. 1952-64, 2009.

[13] H. Sohn et S. Kim, «Development of dual PZT transducers for reference-free crack detection in thin plate structures,» IEEE Trans. Ultrason. Ferroelectr. Freq. Control, vol. 57, p. 229-40, 2010.

[14] E. Lize, M. Rebillat, N. Mechbal et C. Bolzmacher, «Optimal dual-PZT sizing and network design for baseline-free SHM of complex anisotropic composite structures,» Smart Materials and Structures, 2018. 